\title{
Asymptotic Design and Analysis of Multiuser Detector for UWB High Data Rate Channel Based on Channel Division Multiple Access
}

\author{
Houda Chihi, Ridha Bouallegue \\ Higher School of Communications (Sup’Com), Innov’COM, Tunis, Tunisia \\ Email: houda.chihi@gmail.com, ridha.bouallegue@ieee.org
}

Received April 2013

\begin{abstract}
In this paper, we study an innovative multiple access scheme that exploits the intrinsic properties of the wireless environment to improve the multiuser environment, so-called Channel Division Multiple Access (ChDMA) focusing on spectral efficiency analysis and system performance. In particular, we show that Simultaneous multiuser accessing to a common destination is made possible by considering the channel impulse response (CIR) of each user as a signature. We begin by the assumption of the channel state information available at the receiver. Then, we analyze the performance of the ChDMA integration in a random environment over UWB high data rate channel. Next, we discuss the design of MMSE and optimal receiver structure for such a system. Additionally, we show an asymptotic analysis behavior taking into account the channel eigenvalues distribution with the associated spectral efficiency.
\end{abstract}

Keywords: ChDMA; MBOFDM; UWB; Spectral Efficiency; Eigenvalues Distribution; MMSE; Optimal Receiver; PDP

\section{Introduction}

UWB is a promising technology that has attracted considerable interest in the research and standardization communities for wireless communications. For a very large bandwidth, the capacity derivation for UWB channel cannot be the same as for a classical narrowband channel. The main difference results from two frequency dependent attenuation origins: distance and frequency selectivity of channel. Indeed, the attenuation due to distance is proportional to the squared in verse of frequency, and cannot therefore be considered constant over the full UWB bandwidth. Besides, the various realizations of the channel act as linear filters that attenuate randomly the transmitted signal at different frequencies.

In this contribution we describe, firstly, the MBOFDM UWB high data rate system. This must be taken into account in the capacity computation [1]. Next, we present ChDMA Characteristics with the channel model. We shall examine system spectral efficiency in a random environment with channel state information in the recaption. The key performance measure here is in particular the eigenvalue distribution of the channel matrix. Then, in section III, we investigate two receiver structures that we will study in different system behavior: the optimal receiver and the linear minimum mean square error (MMSE) detector. The MMSE linear detector is particu- larly interesting as it allows maximizing the signal-tointerference ratio (SIR) among all linear receivers. In section IV, we present the PDP impact in spectral efficiency. Then, we present the simulation results.

\section{System Description}

\subsection{The Multiband OFDM Solution}

The multiband technique proposed in the WiMedia Alliance MBOFDM scheme divides the UWB spectrum into 14 bands of $528 \mathrm{MHz}$ each, as illustrated in Figure 1. The first 12 bands are then grouped into four band groups consisting of three bands each. The last two bands are grouped into a fifth band group [2]. Initially, most of the studies have been performed on the first three subbands- from 3.1 to $4.8 \mathrm{GHz}$. An OFDM signal can be transmitted on each subband using a 128 point inverse fast Fourier transform (IFFT). Out of the 128 subcarriers used, only 100 are assigned to transmit data. The multiuser access is performed with time frequency codes (TFC) which provide frequency hopping from a subband to another at the end of each OFDM symbol. Hence, at a given instant, if we consider a 3 user system, each user occupies one of the first three subbands [2].

In the MBOA solution case, the signal generated at the output of the IFFT [2] is: 


$$
\begin{aligned}
& S_{\text {OFDM }}(t) \\
& =\sum_{i=-\infty}^{+\infty} \sum_{-M}^{+M} X_{n}(i) p_{c}\left(t-T_{C P}\right) e^{j 2 \pi n \Delta_{F}\left(t-i T_{c p}\right)}
\end{aligned}
$$

$\Delta_{\mathrm{F}}, \mathrm{M}$ and $\mathrm{T}_{\mathrm{CP}}$ represent respectively the subcarriers spacing, the total number of used subcarriers and the spacing between two consecutive OFDM symbols. $\mathrm{X}_{\mathrm{n}}(\mathrm{i})$ is a complex symbol belonging to QPSK constellation and is transmitted by subcarrier $n$ during the ith OFDM symbol. It represents a data, a pilot or a reference symbol, $\mathrm{p}_{\mathrm{c}}(\mathrm{t})$ is a rectangular window defined by [2] as:

$$
\begin{cases}p_{c}(t)=1 & \text { for } 0 \leq t \leq T_{F F T} \\ p_{c}(t)=0 & \text { for } T_{F F T} \leq t \leq T_{F F T}+T_{C P}+T_{G I}\end{cases}
$$

Transmitted data rates in each subband vary from 53.3 to $480 \mathrm{Mbit} / \mathrm{s}$, which are listed in Table 1 [2].

\subsection{UWB Channel Modeling}

The proposed model for MBOFDM is 802.15.3a high data rate channel which derived from Saleh Valenzuela model [3]. Mathematically, the impulse response of the multipath model is given by [4]:

$$
h_{k}(t) X_{k} \sum_{z=0}^{Z_{k}} \sum_{b=0}^{B_{k}} \alpha_{k}(z, b) \delta\left(t-T_{k}(z)-(z, b)\right)
$$

where:

$\mathrm{X}_{\mathrm{k}}$ is the lognormal shadowing of the kth channel realization, $\mathrm{T}_{\mathrm{k}}(\mathrm{z})$ is the delay of cluster $\mathrm{z}$, and $\alpha_{\mathrm{k}}(\mathrm{z}, \mathrm{b}), \tau_{\mathrm{k}}(\mathrm{z}, \mathrm{b})$ represent respectively the gain and the delay of multipath $\mathrm{b}$ withincluster $\mathrm{z}$ respectively.

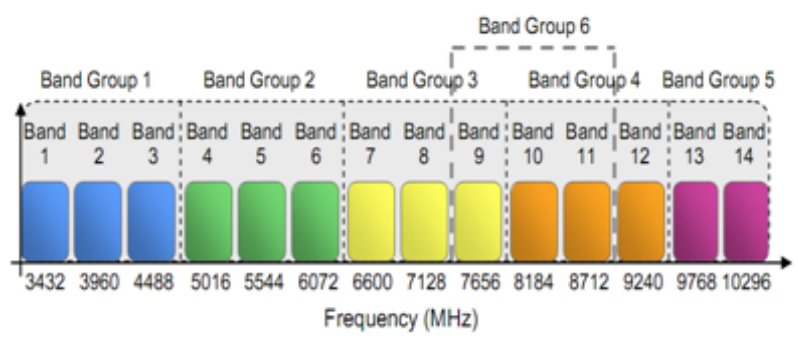

Figure 1. UWB spectrum bands in the MBOFDM system.

Table 1. MBOFDM UWB data rates [4].

\begin{tabular}{cc}
\hline Data rate & Coding rate \\
\hline 53,3 & $1 / 3$ \\
80 & $1 / 2$ \\
106,7 & $1 / 3$ \\
160 & $1 / 2$ \\
200 & $5 / 8$ \\
320 & $1 / 2$ \\
400 & $5 / 8$ \\
480 & $3 / 4$ \\
\hline
\end{tabular}

\subsection{ChDMA-UWB System Principle Analysis}

The idea behind ChDMA is to use the channel impulse response (CIR) of each user as a signature, i.e., the signature code is given by the channel and the users are separated as a CDMA system. It is important to note that the signatures are given by the environment and by user's position, which means that they are uniquely determined. This signature location dependent property provides decentralized flexible multiple access as the codes are naturally generated by the radio channel [5]. The previous multiple access schema don't benefit of using low duty cycle transmissions. For this reason, in this paper we consider a multiuser communications system which exploits the duty cycle assumption and sends a modulated peaky signal. The new multiple access considered system is based on the integration of ChDMA in the 802.15.3a model. The ChDMA proposition works because UWB channels have a large coherence time (typically about $100 \mu \mathrm{s}$ ) relatively to their delay spread (typically around 15 - 40 ns, depending on the user environment) [6]. Following the clustered ray model, each ray is considered as a single replica of the signal due to the distortion accompanied the environment, yields that each user occupies an appropriate position as presented in Figure 2 [6], where we consider an uplink channel between user $\mathrm{K}$ and the destination. For this reason, the transmitted signal identification is easily obtained where the receiver benefits of the different position occupied by each user independently, as it knows the channel it will be able to detect and to demodulate the received waveform.

\section{Spectral Efficiency Performance Analysis}

We analyze the performance of ChDMA UWB system in terms of spectral efficiency using two different receivers:

- The optimal receiver.

- The minimum mean square error receiver.

The spectral efficiency $\gamma$ is defined as the number of bits per chip summed over the users that can be reliably

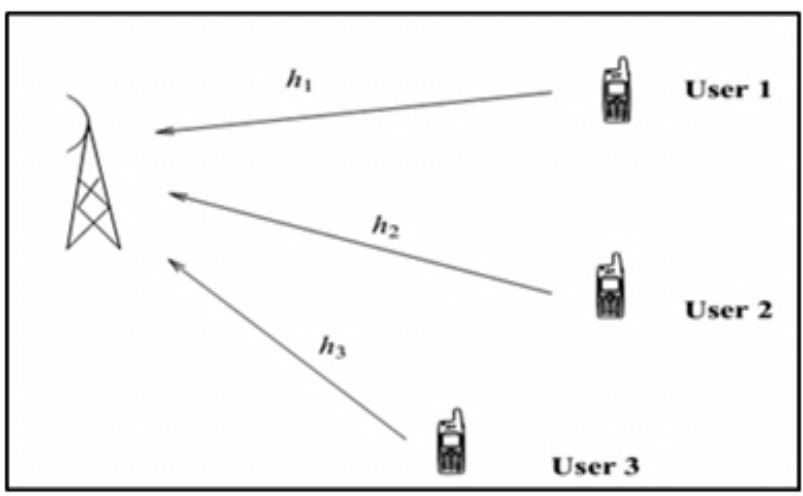

Figure 2. Channel Impulse Division Multiple Access with three users. 
transmitted [7]. It is expressed as the bits per second per Hertz (bit/s/Hz) supported by the system.

\subsection{Linear Minimum Mean Square Error Receiver}

The concept of MMSE detector originates from turning the problem of detection of transmitted symbols in a CDMA system into a problem of linear estimation [8]. In the following, we consider the uplink communication, where the $\operatorname{SINR}_{\mathrm{k}, \mathrm{n}}$ at the output of the MMSE detector is given by [9]:

$$
\operatorname{SINR}_{k, n}=h_{k, n}^{H}\left(H_{k, n} H_{k, n}^{H}+\sigma^{2} I\right)^{-1} h_{k, n} .
$$

where $\mathrm{n}$ is related to nth symbol from $\mathrm{N}$ and $\mathrm{k}$ related to the kth user from $\mathrm{K}, \mathrm{H}_{\mathrm{k}, \mathrm{n}}$ is the matrix obtained from $\mathrm{H}$ suppressing the column $h_{k, n}$. The instantaneous spectral efficiency is given by [5]:

$$
\begin{aligned}
\gamma & =\frac{1}{N} \sum_{i=1}^{N} \log _{2}\left(1+\operatorname{SINR}_{k, n}\right) \\
& =\frac{1}{N} \sum_{i=1}^{N} \log _{2}\left(1+h_{k, n}^{H}\left(H_{k, n} H_{k, n}^{H}+\sigma^{2} I\right)^{-1} h_{k, n}\right) .
\end{aligned}
$$

We must mention that the signal to noise ratio $1 / \sigma^{2}$ is related to the spectral efficiency $\gamma$ following the author in [7] by:

$$
\frac{1}{\sigma^{2}}=\frac{N}{K} \gamma \frac{E_{b}}{N_{o}} .
$$

Deeper insight on the linear MMSE spectral efficiency performance behavior is obtained byK, $\mathrm{N} \rightarrow \infty$ with constant ratio $\beta$ where $\beta=\mathrm{K} / \mathrm{N}$.

The study of the eigenvalue distribution of random matrices has triggered important consideration in many numerous works. For this reason, the performance of MBOFDM ChDMA UWB communication system is determined via the eigenvalues of the covariance matrix $\mathrm{C}_{\mathrm{N}}=\mathrm{H}_{\mathrm{N}} \mathrm{H}_{\mathrm{N}}^{\mathrm{H}}$. We study the eigenvalues distribution performance of linear MMSE multiuser receiver in random environment. The eigenvalue distribution is described in Figure 3 [10]:

The formula for the MMSE demodulator could be described via considering the transformation of $\mathrm{C}_{\mathrm{N}}$ using the singular value decomposition (SVD). We denote the SVD representation of the channel correlation matrix $\mathrm{C}_{\mathrm{N}}$ :

$$
C_{N}=S D S^{T} \text {. }
$$

where $S$ is a unitary matrix containing the eigenvectors of $\mathrm{HH}^{\mathrm{H}}$ and $\mathrm{D}$ is a diagonal matrix, containing the singular values $\lambda_{\mathrm{i}}$ with $\mathrm{i}=1 \cdots \mathrm{N}$ representing the eigenvalues of $\mathrm{HH}^{\mathrm{H}}$. Second, following results from random matrix theory [11], it can be deduced that the empirical distribution of the eigenvalues of $C_{N}$ converges to some limiting distribution $\mathrm{F}$, we obtain:

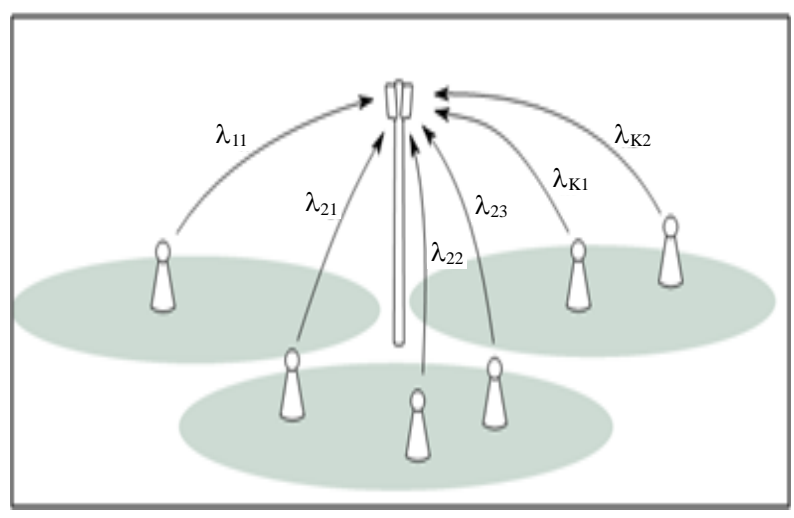

Figure 3. Eigenvalue distribution.

$$
\operatorname{SINR}_{k, n}=h_{k, m}^{H}\left(\lambda_{i}+\sigma^{2} I\right)^{-1} h_{k, n}
$$

We assume a large system, i.e., we let $\mathrm{K}, \mathrm{N} \rightarrow \infty$ while $\mathrm{K} / \mathrm{N}$ is finite and converges to a specific value $\beta$. Yields in the asymptotic analysis, the spectral efficiency can be expressed in terms of the eigenvalue distribution of $\mathrm{HH}^{\mathrm{H}}$ and it is given by:

$$
\Upsilon_{m m s e}=1 / N \sum_{i=1}^{K} \log _{2}\left(1+\frac{\lambda_{i}}{\lambda_{i}+\sigma^{2}}\right) .
$$

We must mention that in [12] (Lemma 9) if we consider the key performance criterion as SIR of user $\mathrm{k}$ under the MMSE detector we obtain:

$$
\sum_{i=1}^{K} \frac{S I R_{k}}{1+S I R_{k}}=\sum_{i=1}^{K} \frac{\lambda_{k}}{1+\lambda_{k}} .
$$

By this way (6) could be described by:

$$
\Upsilon_{\text {mmse }}=1 / N \sum_{i=1}^{K} \log _{2}\left(1+\frac{S I R_{i}}{S I R_{i}+\sigma^{2}}\right) .
$$

We should point out that (6) converges in probability to:

$$
\Upsilon_{\text {mmse }}=\int \log _{2}\left(1+\frac{\lambda_{i}}{\lambda_{i}+\sigma^{2}}\right) f F_{H}^{N}(\lambda) .
$$

where $F_{H}^{N}$ defined by the author in [13] by: $\mathrm{R} \rightarrow \mathrm{R}$ is the empirical eigenvalue distribution (e.e.d) of the random matrix $\mathrm{H}$, i.e.

$$
F_{H}^{N}(\lambda)=\frac{1}{N} \cdot\left|\left\{\lambda_{i}: \lambda_{i} \leq \lambda\right\}\right| .
$$

Let $\mathrm{G}_{\mathrm{A}}(\mathrm{z})$ the Stieltjes Transform of $F_{H}^{N}$ as defined by [14]:

$$
G_{A}(z)=\int \frac{1}{\lambda-z} d F_{H}^{N}(\lambda) \quad \text { for } z \in \mathbb{C}^{+} .
$$

where $\mathbb{C}^{+}=\{z \in \mathbb{C} \mid \operatorname{Im}[z]>0\}$

Yields: 


$$
\begin{aligned}
\Upsilon_{\text {mmse }} & =\int_{0}^{\infty} \log _{2}\left(\frac{\lambda_{i}+\sigma^{2}+\lambda_{i}}{\lambda_{i}+\sigma^{2}}\right) d F_{H}^{N}(\lambda) \\
& =\int_{0}^{\infty} \log _{2}\left(2 \lambda+\sigma^{2}\right) d F_{H}^{N}(\lambda) \\
& -\int_{0}^{\infty} \log _{2}\left(\lambda+\sigma^{2}\right) d F_{H}^{N}(\lambda) .
\end{aligned}
$$

Following Theorem 6 in [15], we have that F converge if $N \rightarrow \infty$ to a distribution function $\mathrm{G}$, we obtain the SNR of the MMSE receiver convergence to:

$$
h_{k, m}^{H}\left(H_{k, m} H_{k, m}^{H}+\sigma^{2} I\right)^{-1} h_{k, m} \rightarrow \rho=\int \frac{1}{\lambda+\sigma^{2}} d G(\lambda) .
$$

Finally we obtain: $\gamma_{\infty} \rightarrow \int \log _{2}(1+\rho) \mathrm{dG}(\lambda)$.

Additionally, for asymptotically large random matrices such upper bounds are provided by random matrix theory. If the channel matrix is composed of independent identically distributed random entries. The eigenvalues of $\mathrm{HH}^{\mathrm{H}}$ are asymptotically upper bounded by [16]:

$$
\lambda_{k}<(1+\sqrt{\beta})^{2}
$$

By this way the bounds of (8) are given by:

$$
\begin{gathered}
\int_{0}^{\infty} \log _{2}\left(2 \lambda+\sigma^{2}\right) \mathrm{dG}(\lambda)<\int \log _{2}\left(2(1+\sqrt{\beta})^{2}+\sigma^{2}\right) \mathrm{dG}(\lambda) \\
\int_{0}^{\infty} \log _{2}\left(\lambda+\sigma^{2}\right) \mathrm{dG}(\lambda)<\int \log _{2}\left((1+\sqrt{\beta})^{2}+\sigma^{2}\right) \mathrm{dG}(\lambda) .
\end{gathered}
$$

Yields:

$$
\begin{aligned}
\Upsilon_{m m s e} & <\int \log _{2}\left(2(1+\sqrt{\beta})^{2}+\sigma^{2}\right) \\
& -\log _{2}\left((1+\sqrt{\beta})^{2}+\sigma^{2}\right) \mathrm{d} G(\lambda)
\end{aligned}
$$

We obtain:

$$
\gamma_{\text {mmse }}<\log _{2} \frac{\left(2(1+\sqrt{\beta})^{2}+\sigma^{2}\right)}{\left((1+\sqrt{\beta})^{2}+\sigma^{2}\right)} .
$$

Following Theorem 2 presented in [17] we have that under the condition of large system the minimal value of eigenvalue is given by:

$$
\lambda_{k}>(1-\sqrt{\beta})^{2} .
$$

The minimum bounds of (8) are given by:

$$
\begin{gathered}
\int_{0}^{\infty} \log _{2}\left(2 \lambda+\sigma^{2}\right) \mathrm{dG}(\lambda)>\int \log _{2}\left(2(1-\sqrt{\beta})^{2}+\sigma^{2}\right) \mathrm{dG}(\lambda) . \\
\int_{0}^{\infty} \log _{2}\left(\lambda+\sigma^{2}\right) \mathrm{dG}(\lambda)>\int \log _{2}\left((1-\sqrt{\beta})^{2}+\sigma^{2}\right) \mathrm{dG}(\lambda) .
\end{gathered}
$$

Let the minimal bound is given by:

$$
\gamma_{m m s e}>\log _{2} \frac{\left(2(1-\sqrt{\beta})^{2}+\sigma^{2}\right)}{\left((1-\sqrt{\beta})^{2}+\sigma^{2}\right)} .
$$

If we consider the case of $0<\beta<1$, and following the author in [18], the density of $\mathrm{F}(\mathrm{x})$ is given by:

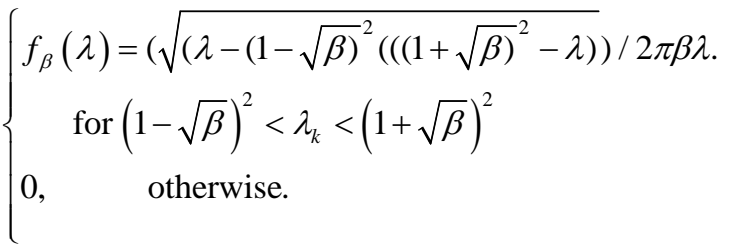

Then, if we consider the case of $0<\beta<\infty$, we obtain:

$$
\left\{\begin{array}{l}
F_{H}^{N}(\lambda)=1-1 / \beta \text { for } 0<\lambda_{k}<(1+\sqrt{\beta})^{2} . \\
F_{H}^{N}(\lambda)=1-1 / \beta+\int_{(1-\sqrt{\beta})^{2}}^{\lambda} f_{\beta}(\lambda) d t \text { for }(1-\sqrt{\beta})^{2}<\lambda_{k}<(1+\sqrt{\beta})^{2} \\
F_{H}^{N}(\lambda)=1 \text { when } \lambda_{k}>(1+\sqrt{\beta})^{2} .
\end{array}\right.
$$

\subsection{Optimal Receiver}

The optimal receiver is defined as the receiver that minimizes the probability of symbol error among all receiver structures. It is based on the analysis of the posterior probabilities of the transmitted signal [19]. The spectral efficiency is defined by:

$$
\gamma=(1 / N) * \log _{2} \operatorname{det}\left(I_{N}+\left(1 / \sigma^{2}\right) H H^{H}\right) .
$$

where: $\sigma^{2}$ is the noise variance and $\mathrm{N}$ the channel vector length. Equation (10) can also be represented like the author in [6]:

$$
\gamma=(1 / N) * \log _{2} \operatorname{det}\left(I_{N}+\rho H H^{H}\right) .
$$

where $\rho$ is defined in [6] by:

$$
\rho=\frac{\gamma}{\beta} \frac{E_{b}}{N o} \text { with } \beta=(\mathrm{K} / \mathrm{N}) .
$$

In fact, the asymptotic analysis allows providing a good understanding of the ChDMA limiting behavior in MBOFDM-UWB channel.

The author in [6] allows us to write the spectral efficiency represented in (10) in terms of the eigenvalues $\left(\lambda_{1} \cdots \lambda_{\mathrm{m}}\right)$ of $\mathrm{HH}^{\mathrm{H}}$ like: 


$$
\gamma=(1 / N) * \sum_{i}^{N} \log \left(1+\rho \lambda_{i}\left(H H^{H}\right)\right) .
$$

Following, the author in [20], when $\mathrm{N} \rightarrow+\infty$, the spectral efficiency can be represented in terms of the eigenvalue distribution of $\mathrm{HH}^{\mathrm{H}}$ yields to:

$$
\gamma_{\infty}=\int \log (1+r \lambda) \mathrm{d} f_{H H}^{H} .
$$

We conclude that the spectral efficiency is deterministic and only depends on a few macroscopic system parameters in asymptotic regime.

\section{Power Delay Profile}

Following the Toeplitz structure of the matrix $\mathrm{C}_{\mathrm{N}}$, the following eigendecomposition holds when $\mathrm{N} \rightarrow \infty$ [21]:

$$
C_{\infty}=\lim _{N \rightarrow \infty} F_{N}^{H} D F_{N} \text {. }
$$

where $F_{N}$ being the Fourier matrix and $D$ could be defined following the decomposition in [22] according to its elements $D_{n, n}$.

where:

PDP refers to power delay profile representing the exponential decay of each cluster; also it reflects the decay of the total cluster power with the delay. The detailed definition is given following the author in [23]:

$$
E\left[\left|\xi_{l} \beta_{k, l}\right|^{2}\right]=\Omega_{0} e^{\frac{-T_{l}}{\Gamma}} e^{\frac{-\tau_{k, l}}{\gamma}} .
$$

with $\Omega_{0}$ represents the average energy of the first path of the first cluster, $\Gamma$ and $\gamma$ are, respectively, defined as constants that characterize the exponential decay of each cluster and each ray.

- $\mathrm{W}_{\mathrm{c}}$ represents the frequency resolution.

- $\mathrm{T}_{\mathrm{l}}$ is the arrival time of the first path of the lth cluster and $\mathrm{L}$ is the number of multipath.

We evaluate the impact of a power delay profile (PDP) on the spectral efficiency. In fact, if we refer to (14) and replace PDP with his previous expression in (15), we can represent the results of spectral efficiency with a random generation of the channel by employing the diagonal matrix D .We assume, respectively, the number of frequency samples $\mathrm{N}$, the frequency resolution $\mathrm{W}_{\mathrm{c}}$, the number of multipath $\mathrm{L}, 128,40 \mathrm{MHz}$ and 100.

Figure 4 presents the impact of the PDP on the spectral efficiency. As the SNR increase, the spectral efficiency of the simulated channel increase and this depend intimately to the system parameters. The result shows that the energy is equally spread over all the bandwidth.
In fact, PDP has been shown to be a useful measure, and we have employed it to identify the optimum number of parameters to represent the performance. This increase of spectral efficiency is also due to the attractive choice of OFDM for UWB communication because it can capture the multipath energy efficiently.

The obtained result shows that the clusters are disjoint because we have an increase of spectral efficiency. However, we can find generally some overlap between the lth and $(l+1)$ th clusters.

\section{Results}

In this section, we present the simulation results obtained by averaging over 500 Monte Carlo iteration, we consider the mode I of the MBOFDM employing the first three subbands of $528 \mathrm{MHz}$. Additionally, each realization of the channel model related to each user is generated independently and assumed to be time invariant during the transmission of a frame. We simulated the MMSE spectral efficiency using the CM1UWB channel model where the parameters are presented in Table 2 [24] which is presented in Figure 5.

In Figure 6 we present a comparison of the spectral efficiency of optimal receiver performance results over the CM1 UWB model. The obtained results show that the performance of the MBOFDM ChDMA-UWB high data rate system is critically dependent of the receiver structure as the number of users increase, the spectral efficiency increases as well. The increase in spectral efficiency comes from the multiuser diversity effect provided by ChDMA system; if we consider many users transmitting independently, we could find a user with strong

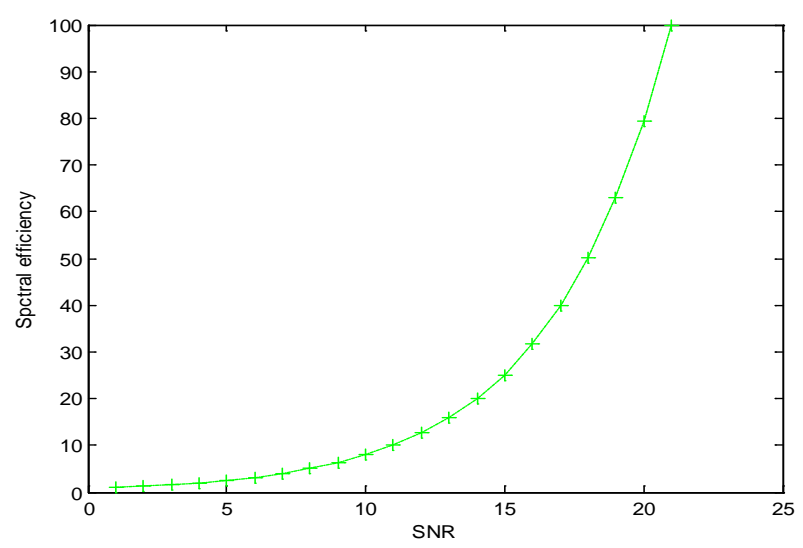

Figure 4. Impact of the power delay profile.

$$
\left\{\begin{array}{l}
\left(\mathrm{PDP}^{*} \mathrm{~L}\right) /\left(\mathrm{W}_{\mathrm{c}} * \mathrm{~T}_{1}\right) \text { with }\left(\mathrm{NW}_{\mathrm{c}} \mathrm{T}_{1}\right) *(\mathrm{l}-1) / \mathrm{L} \leq \mathrm{n}-1 \leq\left(\mathrm{NW}_{\mathrm{c}} \mathrm{T}_{1}\right) * \mathrm{l} / \mathrm{L} \\
\mathrm{F}_{\mathrm{H}}^{\mathrm{N}}(\lambda)=1 ? \quad \beta+\int_{(1-\sqrt{\beta})^{2}}^{\lambda} \mathrm{f}_{\beta}(\lambda) d t \quad \text { for }(1-\sqrt{\beta})^{2}<\lambda_{\mathrm{k}}<(1+\sqrt{\beta})^{2} \\
\mathrm{D}_{\mathrm{n}, \mathrm{n}}=0 . \text { Otherwise }
\end{array}\right.
$$


channel yields that the channel is used in the most efficient manner where the total throughput is maximized.

By this way, we obtain a maximized multiuser diversity gain .We must mention also that QPSK is the best practical choice in the MBOFDM ChDMA-UWB channel coherent regime when the receiver knows the channel.

\section{Conclusions}

In this contribution we aim at providing a very good technical solution to be proposed for UWB physical layer

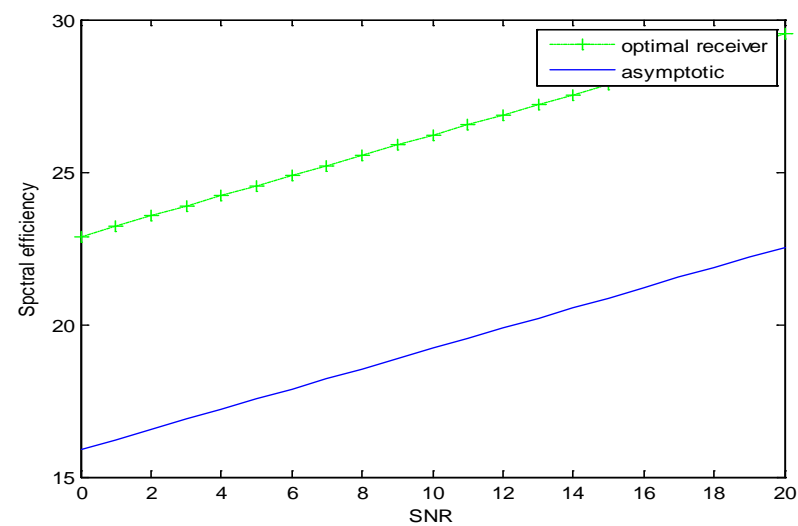

Figure 5. Validation of ChDMA-UWB channel model with MMSE detector $(\mathrm{Eb} / \mathrm{No}=5 \mathrm{~dB})$.

Table 2. MBOFDM UWB system parameters.

\begin{tabular}{cc}
\hline \multicolumn{1}{c}{ WiMedia } & \\
\hline Number of data subcarriers & 100 \\
number of pilot symbols & 12 \\
number of guard symbols & 10 \\
Subcarrier frequency spacing (Mhz) & 4,125 \\
zero padding duration (ns) & 60,61 \\
\hline
\end{tabular}

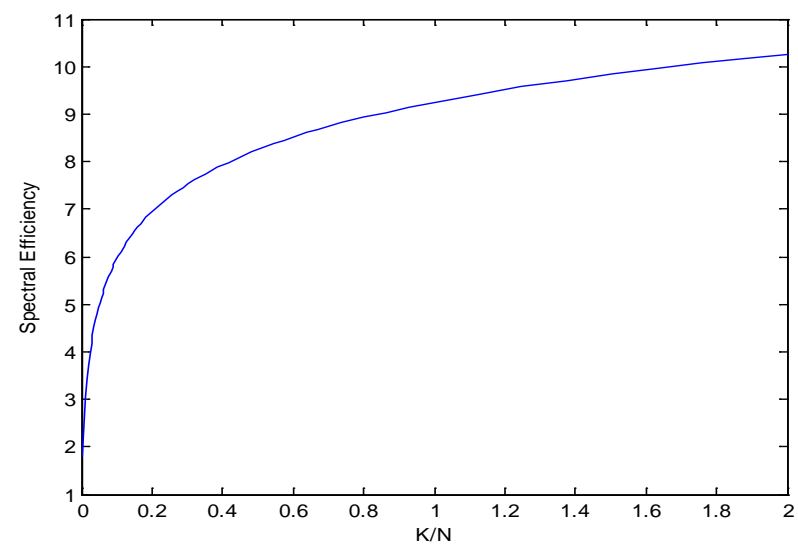

Figure 6. Comparison between optimal and asymptotic spectral efficiency. high data rate wireless applications called ChDMA. This approach, simplifies the transmitter architecture allowing simultaneous access as we assimilate the natural dispersion of the channel to code signature. We have studied the eigenvalues distribution performance analysis of the MMSE and optimal receiver in MBOFDM ChDMAUWB channel in terms of spectral efficiency assuming that the system size is large. Additionally, we investigated the impact of power delay profile (PDP) on the spectral efficiency of MBOFDM UWB-ChDMA high data rate system.

As perspectives, we could investigate other receiver structure that will be adapted for the considered system. We are more ambitious in studying mutual information criteria as well channel estimation mismatches.

\section{REFERENCES}

[1] M. Kamoun, S. Chaillouz, J. Gosteau, L. Mazet and M. de Courville Pierre Duhamel, "Data Rate Upper Bounds for UWB Link with IEEE802.15.3a Channel Model," IEEE International Conference, ICU 2005.

[2] A. Stephan, "Resource Allocation Strategies and Linear Precoded OFDM Optimization for Ultra-Wideband Communications,” Ph.D. Thesis, National Institute of Applied Sciences (INSA), 2008.

[3] A. Saleh and R. Valenzuela, "A Statistical Model for Indoor Multipath Propagation,” IEEE Journal on Selected Areas in Communications, Vol. 5, No 2, 1987, pp. 128137. http://dx.doi.org/10.1109/JSAC.1987.1146527

[4] J. Foerster, "Channel Modeling Sub-Committee Report Final,” IEEE Document P802.15-02/490r1, Intel R\&D, 2003.

[5] Raul L. de Lacerda Neto, Aawatif Menouni Hayar and Merouane Debbah, "Channel Division Multiple Access: New Multiple Access Approach for UWB Networks," European Ultra Wide Band Radio Technology Workshop 2007, Grenoble, France, 10-11 May 2007.

[6] R. L. De LacerdaNeto, "Exploiting the Wireless Channel for Communication,” Ph.D. Thesis, Nice-Sophia Antipolis University, 2008.

[7] S. Verdu and S. Shamai, "Spectral Efficiency of CDMA with Random Spreading," IEEE Transactions on Information Theory, 1999, pp. 622-640. http://dx.doi.org/10.1109/18.749007

[8] Z. Xie, R. T. Short and C. K. Rushforth, "A Family of Suboptimum Detectors for Coherent Multiuser Communications," IEEE Journal on Selected Areas in Communications, Vol. 8, No. 4, 1990, p. 683.

[9] L. Cottatellucci, R. R. Muller, M. Debbah, "On the Capacity of Asynchronous CDMA Systems," Conference Record of the Forty-First Asilomar 2007.

[10] R. Couillet, "Random Matrices in Wireless Communications Course 2: System Performance Analysis: Capacity and Rate Regions,” 2009.

[11] V. A. Marcenko and L. A. Pastur, "Distribution of Eigen- 
values for Some Sets of Random Matrices,” Mathematics of the USSR, Vol. 1, 1967, pp. 457-483. http://dx.doi.org/10.1070/SM1967v001n04ABEH001994

[12] S. V. Hanly and D. N. C. Tse, "Resource Pooling and Effective Bandwidths in CDMA Networks with Multiuser Receivers and Spatial Diversity," IEEE Transactions on Information Theory, Vol. 47, No. 4, 2001.

[13] M. J. M. Peacock, I. B. Collings and M. L. Honig, "Asymptotic Spectral Efficiency of Multiuser Multisignature CDMA in Frequency-Selective Channels," IEEE Transactions on Information Theory, Vol. 52, No. 3, 2006. http://dx.doi.org/10.1109/TIT.2005.864427

[14] R. Wegmann, “The Asymptotic Eigenvalue-Distribution for a Certain Class of Random Matrices," Journal of Mathematical Analysis and Applications, Vol. 56, 1976, pp. 113-132. http://dx.doi.org/10.1016/0022-247X(76)90011-1

[15] J. Evans and D. N. C. Tse, "Large System Performance of Linear Multiuser Receivers in Multipath Fading Channels," IEEE Transactions on Information Theory, Vol. 46, No. 6, 2000. http://dx.doi.org/10.1109/18.868478

[16] R. R. Muller, “Applications of Large Random Matrices in Communications Engineering,” Statistical Mechanics, 2. Spin Glasses, 2003.

[17] Z. D. Bai and Y. Q. Yin, "Limit of the Smallest Eigenvalue of a Large Dimensional Sample Covariance Matrix," Annals of Probability, Vol. 21, No. 3, 1993, pp. 1275-
1294. HTTP://DX.DOI.ORG/10.1214/AOP/1176989118

[18] J. W. Silverstein, "Some Limit Theorems on the Eigenvectors of Large Dimensional Sample Covariance Matrices,” Journal of multivariate Analysis, Vol. 15, 1984, pp. 295-324. http://dx.doi.org/10.1016/0047-259X(84)90054-X

[19] J. Proakis (2000) Digital Communications. McGraw Hill.

[20] I. E. Telatar, "Capacity of Multi-Antenna Gaussian Channels,” Technical Report, AT\&T Bell Laboratories Technical Memorandum, 1995.

[21] R. M. Gray, "Toeplitz and Circulant Matrices: A Review,” Technical Report. No. 6504-1, Information Systems Laboratories: ISL, Stanford University, Stanford, CA, 1977.

[22] R. de Lacerda, L. Cottatellucci, A. Hayar and M. Debbah, "Asymptotic Analysis of Channel Division Multiple Access Schema for Ultra Wideband Channel," 9th IEEE Workshop (SPAWC’08), 2008.

[23] A. Batra, J. Balakrishnan, G. R. Aiello, J. R. Foerster and A. Dabak, "Design of a multiband OFDM System for Realistic UWB Channel Environments," IEEE Transactions on Microwave Theory and Techniques, Vol. 52, 2004, pp. 2123-2138. http://dx.doi.org/10.1109/TMTT.2004.834184

[24] E. Gueguen, M. Crussiere and J.-F. Helard, "An OFDMCDMA Scheme for High Data Rate UWB Applications," VTC Spring 2007, pp. 2905-2909. 